\title{
OPTIMIZACIÓN DE PARÁMETROS PARA LA CONSTRUCCIÓN DE LA CURVA DE RUPTURA EN LA ADSORCIÓN DE Cr(VI) SOBRE CÁSCARA DE CACAO
}

\section{PARAMETER OPTIMIZATION FOR Cr(VI) ADSORPTION BREAKDOWN CURVES ONTO COCOA SHELL}

\author{
Candelaria Tejada-Tovar ${ }^{1}$, Ángel Villabona-Ortíz ${ }^{2}$, Victoria Caballero Romero ${ }^{3}$, Juan Paternina Cuesta ${ }^{4}$, \\ Clemente Granados Conde ${ }^{5}$
}

\begin{abstract}
${ }^{1}$ M.Sc. Ingeniería Ambiental, M.Sc. Educación, Ingeniera Química. Universidad de Cartagena, Grupo de Investigación en Diseño de Procesos y Aprovechamiento de Biomasas-IDAB. Avenida del Consulado Calle 30 No. 48-152, Cartagena - Bolívar, Colombia, e-mail: ctejadat@unicartagena.edu.co; ${ }^{2}$ M.Sc. en Ingeniería Ambiental, Especialista en Ingeniería Sanitaria y Ambiental, Ingeniero Químico. Universidad de Cartagena, Grupo de Investigación en Diseño de Procesos y Aprovechamiento de Biomasas-IDAB. Avenida del Consulado Calle 30 No. 48-152, Cartagena - Bolívar, Colombia, e-mail: avillabonao@unicartagena.edu.co; ${ }^{3}$ Ingeniera química. Universidad de Cartagena, e-mail: vcaballeror@unicartagena.edu.co; ${ }^{4}$ Ingeniero Químico. Universidad de Cartagena, e-mail: jpaterninac@unicartagena.edu.co; ${ }^{5}$ M.Sc. en Ciencia y Tecnología de alimentos, Ingeniero de Alimentos. Universidad de Cartagena, Grupo INCAS. Avenida del Consulado Calle 30 No. 48-152, Cartagena - Bolívar, Colombia, e-mail: cgranados@cunicartagena.edu.co
\end{abstract}

Rev. U.D.C.A Act. \& Div. Cient. 21(1): 167-177, Enero Junio 2018

https://doi.org/10.31910/rudca.v21.n1.2018.675

\section{RESUMEN}

El objetivo de la presente investigación fue evaluar la cáscara de la mazorca cacao (Theobroma cacao L.) para su uso en la adsorción de cromo hexavalente en solución acuosa, usando columna de lecho empacado, aplicando el método de superficie de respuesta (MSR), con el fin de determinar los valores óptimos de las variables incidentes en el proceso $y$, posteriormente, con estos valores, realizar los ensayos de adsorción. Se utilizó un diseño de experimentos compuesto central con puntos estrellas. La biomasa, se lavó, se secó, se disminuyó tamaño y se tamizó; los experimentos, se llevaron a cabo a $25^{\circ} \mathrm{C}$, a diferentes tamaños de partícula, de caudal y de cantidad de biomasa, en columnas en sistema en continuo, obteniéndose un porcentaje de remoción de $39,16 \%$, de $\mathrm{Cr}(\mathrm{VI})$. Al realizar los experimentos en sistema por lotes, se obtuvo que a tamaño de partícula $0,355 \mathrm{~mm}$, se favorece la adsorción. De los ensayos de adsorción en columna, utilizando los parámetros óptimos arrojados obtenidos por el MSR, se obtuvo un porcentaje de remoción de 62,65\%, encontrando que los modelos de Yoon-Nelson y Thomas fueron los que ajustaron los datos experimentales de la curva de ruptura, con $R^{2}=0,98$. Se concluye que la cáscara de cacao es un buen precursor de bioadsorbentes y que las variables más influyentes en la adsorción de $\mathrm{Cr}(\mathrm{VI})$ en sistema por lotes y continuo son el tamaño de partícula y la altura del lecho.
Palabras clave: Biomasa; columna lecho empacado; método superficie respuesta

\section{SUMMARY}

The aim of the present investigation was to evaluate the cocoa shell (Theobroma cacao L.) for its use in the adsorption of hexavalent chromium in aqueous solution using packed bed column, applying the surface response method (SRM) in order to determine the optimum values of the variables involved in the process. The adsorption tests were carried out with these values. Was used a central composite experiment design with star points. The biomass was washed, dried, decreased size and sieved; the experiments were carried out at $25^{\circ} \mathrm{C}$, at different particle sizes, flow rate and amount of biomass, in columns in continuous system; obtaining a percentage of removal of $39.16 \%$ of $\mathrm{C}(\mathrm{VI})$. Was obtained when performing the experiments in batch system that at a size of $0.355 \mathrm{~mm}$ particle favors the adsorption. From the adsorption tests in the column using the optimum parameters obtained by the MSR, was obtained a removal percentage of $62.65 \%$; finding that the models of Yoon-Nelson and Thomas were those that adjusted the experimental data of the rupture curve, with R2 = 0.98. It's concluded that the cocoa shell is a good precursor of bioadsorbents and that the most influential variables in $\mathrm{Cr}$ (VI) adsorption in batch and continuous systems are particle size and bed height. 
Key words: Biomass, fixed bed column, chromium hexavalent, response surface method.

\section{INTRODUCCIÓN}

El cromo hexavalente, generalmente, está presente en el vertido de aguas provenientes de curtiembres, de cromado, de niquelado, de fábricas de colorante, de pigmentos, entre otros, siendo un contaminante que tiene efectos adversos en la salud y en la biota acuática (Mishra \& Bharagava, 2015; Taghipour \& Jalali, 2015; Singh et al. 2015). Para su remoción, se han utilizado diferentes métodos fisicoquímicos, como la precipitación química, el intercambio iónico, entre otros; es así, como la adsorción con biomasas residuales resulta ser un proceso atractivo, por los bajos costos de su implementación tecnológica y la disponibilidad de materias primas, que han demostrado alta eficiencia en la remoción de metales pesados (Tejada-Tovar et al. 2015a; 2016a).

En la remoción de estos metales en sistemas por lotes y continuo, se han usado biomasas residuales, a partir de tuza de maíz y cáscara de naranja, de acuerdo con Tejada-Tovar et al. (2015b); Fumaria indica, como lo reportan Iqbal \& Khera (2015); cáscaras de ñame y de limón, en Tejada-Tovar et al. (2015c); Cystoseira crinitophylla, usado por Christoforidis et al. (2015); turba de coco, probado por Vijayaraghavan et al. (2016) y cáscara de cacao, evaluada por Lara et al. (2016). En este mismo contexto, para la remoción de $\mathrm{Cr}$ (VI) en aguas residuales, se ha usado carbones activados, a partir de fibra de kernel de palma, reportado por Kundu et al. (2014); de semillas de aguaje, descrito por Sun-Kou et al. (2014) y el evaluado por Di Natale et al. (2015); asimismo, se usaron diferentes tipos de cáscaras, tales como nuez de macadamia, obtenido por Pakade et al. (2016); Pleurotus mutilus, ensayada por Alouache et al. (2017); calabaza blanca, probada por Sreenivas et al. (2014); cacao pretratada con ácido clorhídrico e hidróxido de sodio, evaluada por Tejada-Tovar et al. (2017a); Vigna subterranea (L.) Verdc, según Nharingo et al. (2016); naranja, según Tejada-Tovar et al. (2015d), entre otras, obteniendo porcentajes de remoción satisfactorios, mostrando que las biomasas residuales son buenos precursores de adsorbentes de $\mathrm{Cr}(\mathrm{VI})$ en solución.

El cacao es un producto agroalimentario y sus exportaciones en grano han representado más del $71 \%$ de volumen producido. En la explotación cacaotera solo se aprovecha económicamente la semilla, que representa alrededor de un $10 \%$ del peso del fruto fresco; en Colombia, en 2016, se produjeron 107.891,5Ton de cacao, de acuerdo con los informes de la Federación Nacional de Cacaoteros-Fedeacacao (Fedecacao, 2016). La generación de esta gran cantidad de residuos genera problemas ambientales, como la aparición de olores y problemas de disposición; constituyéndose en un vector para la propagación del hongo de especie Phyto- phthora, atacando directamente la productividad del cultivo. El propósito de esta investigación fue evaluar la cáscara de cacao para su uso en la adsorción de $\mathrm{Cr}(\mathrm{VI})$, en columna de lecho empacado, utilizando el método de superficie de respuesta (MSR), para determinar los valores óptimos de las variables incidentes en el proceso.

\section{MATERIALES Y MÉTODOS}

Para la realización de la presente investigación, se tomaron, como variables independientes, el $\mathrm{pH}$, la dosis de adsorbente en $1 \mathrm{~L}$ de solución $(\mathrm{g} / \mathrm{L})$, el tamaño de partícula $(\mathrm{mm})$, la cantidad de biomasa $(\mathrm{g})$ y el caudal; como variable dependiente, la capacidad de adsorción de $\mathrm{Cr}(\mathrm{VI})(\mathrm{g} \mathrm{Cr}(\mathrm{VI}) / \mathrm{g}$ adsorbente) y como variables intervinientes, la temperatura $\left({ }^{\circ} \mathrm{C}\right)$ y la velocidad de agitación (rpm). Se estudió la influencia del tamaño de partícula en sistema por lotes en la remoción de $\mathrm{Cr}(\mathrm{VI})$ sobre cáscara de cacao, como bioadsorbente, siguiendo el diseño experimental factorial multivariado, en el cual, se consideraron 3 niveles de tamaño de partícula $(0,355 \mathrm{~mm}$, $0,5 \mathrm{~mm}, 0,1 \mathrm{~mm})$, a pH 2. Asimismo, se evaluó el efecto de la concentración del adsorbente, tamaño de partícula y caudal, a temperatura constante en la capacidad de remoción de $\mathrm{Cr}$ (VI) sobre el mismo bioadsorbente, siguiendo el diseño compuesto central con puntos estrellas, mostrado en la tabla 1. La concentración del ion en cada muestra, se determinó por espectroscopia UV-vis a 540nm, mediante la reacción de los iones, con 1,5-difenilcarbazida (250mg,) con acetona (50mL), usando agua desionizada, como blanco, en un espectofotómetro UV-Vis Shimadzu UV 1700 (Tejada-Tovar et al. 2016c; 2017).

Materiales y equipos. Para la realización de la etapa experimental, se usaron los siguientes equipos: incubadora, tipo Shaking incubator IN-666; tamizadora, tipo Shaker; molino de cuchillas, medidor de $\mathrm{pH} / \mathrm{iones}$ y balanza analítica $0,001 \mathrm{~g}$; todos los reactivos químicos usados en el presente estudio fueron de grado analítico marca Merk. Los experimentos, se realizaron por duplicado, bajo las mismas condiciones, para mayor confiabilidad de los datos.

Preparación de la biomasa. El biomaterial fue lavado con agua destilada, con el fin de retirar compuestos solubles y eliminar suciedades e impurezas, tales como taninos, resinas azúcares reductores y agentes colorantes. Posteriormente, se secó en una mufla a $95^{\circ} \mathrm{C}$, durante $24 \mathrm{~h}$ (Tejada-Tovar et al. 2015e). A continuación, se redujo de tamaño en un molino de cuchillas; la clasificación, se hizo en una tamizadora tipo Shaker, con tamaños de malla 0,355, 0,5, 1, 1,7, 2,36, 3,35, 4,75 y 6,3mm (Han et al. 2016).

Preparación de soluciones sintéticas. Las soluciones fueron preparadas añadiendo $0,707 \mathrm{~g}$ de $\mathrm{K}_{2} \mathrm{Cr}_{2} \mathrm{O}_{7}$ a $500 \mathrm{~mL}$ de agua desionizada, obteniendo una concentración de 500ppm; lue- 
Tabla 1. Diseño experimental para la adsorción de Cr(VI) en sistema continuo.

\begin{tabular}{|c|c|c|}
\hline Tamaño de Partícula (mm) & Cantidad de Biomasa (g) & Caudal $(\mathrm{mL} / \mathrm{s})$ \\
\hline 1,70 & 30,0 & 0,75 \\
\hline \multirow{4}{*}{2,362} & 20,0 & \multirow{2}{*}{0,5} \\
\hline & 40,0 & \\
\hline & 20,0 & \multirow{2}{*}{1} \\
\hline & 40,0 & \\
\hline \multirow{6}{*}{3,35} & 13,18 & \multirow{4}{*}{0,75} \\
\hline & 30,0 & \\
\hline & 30,0 & \\
\hline & 46,82 & \\
\hline & \multirow{2}{*}{30,0} & 0,33 \\
\hline & & 1,17 \\
\hline \multirow{4}{*}{4,750} & 20,0 & \multirow{2}{*}{0,5} \\
\hline & 40,0 & \\
\hline & 20,0 & \multirow{2}{*}{1} \\
\hline & 40,0 & \\
\hline 6,30 & 30,0 & 0,75 \\
\hline
\end{tabular}

go, se tomó $20 \mathrm{~mL}$ de la solución patrón y se aforó hasta 100mL con la concentración deseada, de 100ppm (Wu et al. 2013; Tejada-Tovar et al. 2017).

Ensayos de adsorción en sistema por lotes. Para determinar el mejor tamaño de partícula, se colocó, en contacto, el biomaterial con la solución del metal, a $100 \mathrm{ppm}$, a $25^{\circ} \mathrm{C}$ y pH 2, durante 120min, con relación biomasa/solución de $5 \mathrm{~g} / \mathrm{L}$. La capacidad de adsorción, se calculó mediante la fórmula:

$$
\mathrm{q}_{\mathrm{e}}(\mathrm{mg} / \mathrm{m})=\frac{\mathrm{V}\left(\mathrm{C}_{\mathrm{o}}-\mathrm{C}_{\mathrm{f}}\right)}{\mathrm{M}}
$$

Donde, qe es la capacidad de adsorción en el equilibrio en $\mathrm{mg}$ de metal adsorbido/masa en $\mathrm{g}$ de adsorbente; $\mathrm{C}_{\mathrm{y}} \mathrm{y} \mathrm{C}_{\mathrm{f}}$ son las concentraciones iniciales y finales en el equilibrio $(\mathrm{mg} / \mathrm{L})$; $V$ es el volumen de la solución (L) y M, la masa de adsorbente usado (g) (Tejada-Tovar et al. 2016b).

Cinética e isotermas de adsorción. El estudio cinético, se realizó durante $270 \mathrm{~min}$, tomando una alícuota de $5 \mathrm{~mL}$, cada $30 \mathrm{~min}$, entre 10 y $270 \mathrm{~min}$, ajustando los datos experimentales de adsorción a los modelos de primer orden, segundo orden, Elovich y Difusión intraparticular (Tabla 2), con el fin de determinar el mecanismo de adsorción. El estudio de equilibrio de adsorción, se realizó usando diferentes concentraciones iniciales de contaminante en la solución: 25, 50, 75 y $100 \mathrm{pm}$, durante $60 \mathrm{~min}$, sometiendo los datos experimen- tales a los modelos de isoterma de Langmuir y Freundlich (Tabla 2).

Ensayos de biosorción en sistema continuo. Se llevaron a cabo en un equipo que contempla flujos descendentes a tasa constante, nivel constante y por gravedad, para tener un mejor control y manejo hidráulico de la planta y disminuir los gastos energéticos asociados a su funcionamiento; consta de dos lechos (Figura 1), con diámetro de $4,7 \mathrm{~cm}$ y altura de $53 \mathrm{~cm}$; dispone de 12 válvulas, para controlar el flujo; un tanque de almacenamiento de 8,8L; una bomba periférica, para la distribución de alimentación de las soluciones metálicas a 100ppm; un tanque de recolección de 5L y dos tanques de distribución de $2 \mathrm{~L}$. Se utilizaron columnas acrílicas, donde se colocó el lecho de biomasa tratada, variando la cantidad de bioadsorbente, con el fin de tener diferentes alturas de lecho. La solución con el contaminante se bombeó, usando cinco caudales diferentes, durante $1 \mathrm{~h}(0,33,0,5,0,75,1$, $1,17 \mathrm{~mL} / \mathrm{s})$, teniendo en cuenta el diseño de la tabla 1 . Para la construcción de la curva de ruptura, se utilizaron los valores óptimos de tamaño de partícula, caudal y cantidad de biomasa, calculados con el software Statgraphics Centurión XVI.I y se tomaron muestras cada $30 \mathrm{~min}$, durante $5 \mathrm{~h}$, que conllevó a un valor de respuesta óptima, mediante la MSR, obteniendo así una ecuación de regresión, que ajustó todos los datos del sistema, siendo la variable de respuesta el porcentaje de remoción de $\mathrm{Cr}(\mathrm{VI})$. 


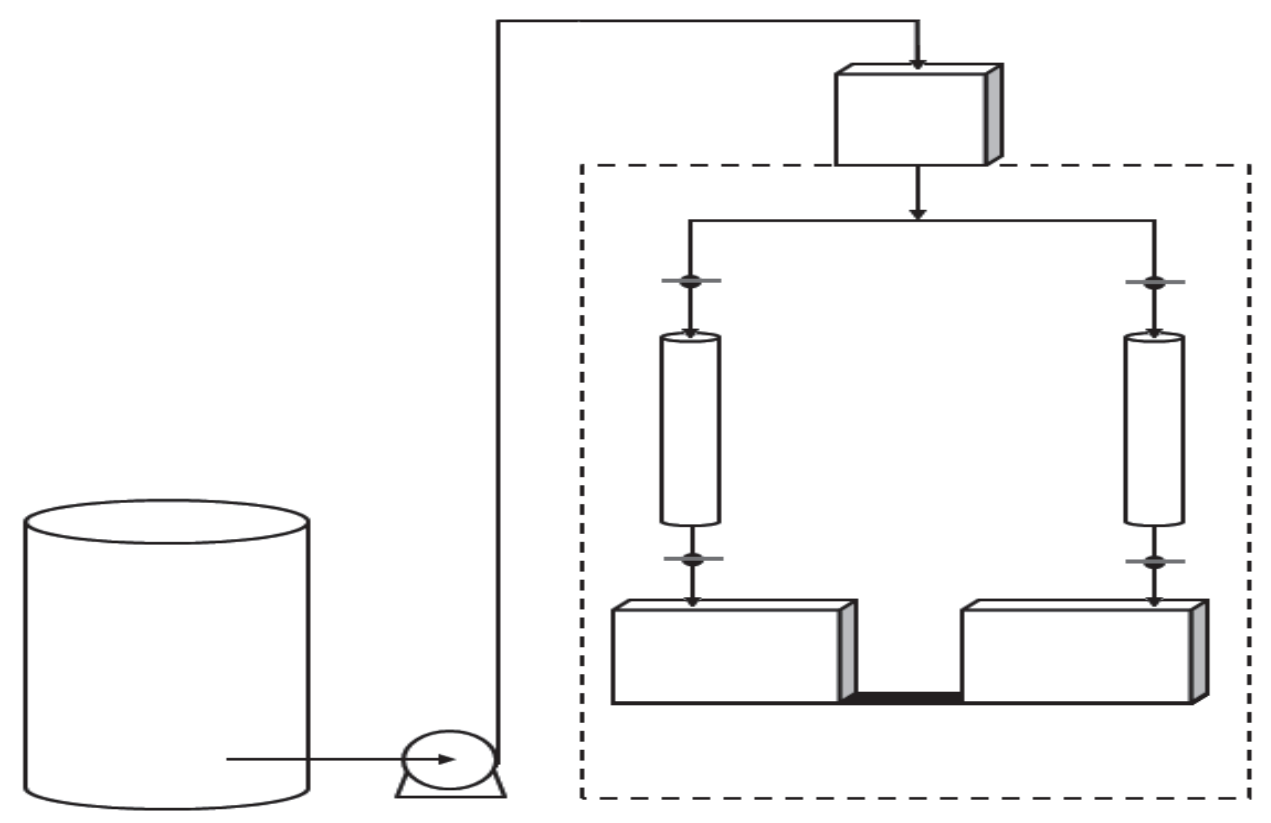

Figura 1. Esquema de equipo en sistema continuo.

Fuente: Tejada-Tovar et al. 2017b.

Tabla 2. Modelos de ajuste de los datos experimentales de adsorción de $\mathrm{Cr}(\mathrm{VI})$ sobre cáscara de cacao en sistema por lotes y continuo.

\begin{tabular}{|c|c|c|}
\hline Modelo cinético & Ecuación & Parámetros \\
\hline Primer orden & $q_{t}=q_{e}\left(1-e^{-k t}\right)$ & $\begin{array}{l}\text { qe Capacidad de adsorción en } \\
\text { equilibrio }(\mathrm{mmol} / \mathrm{g}), \mathrm{k} \\
\text { constante de psudo primer } \\
\text { orden (min- } 1 \text { ) }\end{array}$ \\
\hline Segundo orden & $q_{t}=\frac{t}{\left(\frac{1}{K_{2} q_{e}^{2}}\right)+\left({ }^{t} / q_{e}\right)}$ & $\begin{array}{l}\text { k constante de pseudo } \\
\text { segundo orden ( } \mathrm{g} / \mathrm{mmol} . \mathrm{min}) \text {, } \\
\text { qe Capacidad de adsorción en } \\
\text { equilibrio (mmol/g) }\end{array}$ \\
\hline Elovich & $q t=\frac{1}{\beta} \ln (\alpha \beta)+\frac{1}{\beta} \ln t$ & $\begin{array}{l}\alpha \text { Constantes de Elovich } \\
\text { (mmol/g.min), } \beta \text { Exponente } \\
\text { en la ecuación de Elovich } \\
(\mathrm{g} / \mathrm{mmol})\end{array}$ \\
\hline Difusión Intraparticular & $q_{t}=k \sqrt{t}$ & $\mathrm{~K}$, constante de difusión \\
\hline Modelo de isoterma & Ecuación & Parámetros \\
\hline Langmuir & $q_{e}=q \max \frac{b C_{e}}{1+b C_{e}}$ & $\begin{array}{l}\text { qe es la concentración del } \\
\text { metal adsorbido en el } \\
\text { adsorbente, } C_{\mathrm{e}} \text { es la } \\
\text { concentración residual del } \\
\text { metal en solución, qmax es la } \\
\text { adsorción máxima, b es la } \\
\text { relación entre la tasas de } \\
\text { adsorción/desorción }\end{array}$ \\
\hline
\end{tabular}


Continuación Tabla 2.

\begin{tabular}{|c|c|c|}
\hline Freundlich & $q_{e}=K_{f} C_{e}^{1 / n}$ & $\begin{array}{l}\mathrm{K}_{\mathrm{f}} \text { es la constante de } \\
\text { Freundlich, } \mathrm{n} \text { es la intensidad } \\
\text { de adsorción, } \mathrm{q}_{\mathrm{e}} \text { es la } \\
\text { cantidad de metal adsorbido } \\
\text { en el equilibrio, } \mathrm{C}_{\mathrm{e}} \text { es la } \\
\text { concentración residual del } \\
\text { metal en solución }\end{array}$ \\
\hline $\begin{array}{l}\text { Modelo de curva de } \\
\text { ruptura }\end{array}$ & Ecuación & Parámetro \\
\hline Adams-Bohar & $\frac{C}{C_{i}}=e^{k_{A B} C_{i} t-\frac{k_{A B} N_{0} Z}{v}}$ & $\begin{array}{l}C \text { concentración de soluto } \\
(\mathrm{mg} / \mathrm{L}), k_{A B} \text { cte }(\mathrm{L} / \mathrm{mgmin}), N_{0} \\
\text { capacidad de sorción máxima } \\
\text { (mg/L), } Z \text { altura del relleno de } \\
\text { la columna }(\mathrm{cm}), v \text { velocidad } \\
\text { de flujo lineal }(\mathrm{cm} / \mathrm{min})\end{array}$ \\
\hline Thomas & $\frac{C}{C_{i}}=\frac{1}{1+e^{\left(\frac{k_{T h}}{Q}\left(q_{\max } w-C_{i} V_{e f}\right)\right)}}$ & $\begin{array}{l}k_{T h} \text { constante de velocidad } \\
\text { (L/mgmin), } V_{e f} \text { caudal }\end{array}$ \\
\hline Yoon-Nelson & $\frac{C_{i}}{C}=\frac{1}{1+e^{k_{Y N}(\tau-t)}}$ & $\begin{array}{l}\tau \text { tiempo requerido para } \\
\text { retener el } 50 \% \text { del adsorbato } \\
\text { inicial (min), } k_{Y N} \text { cte de } \\
\text { proporcionalidad }\left(\mathrm{min}^{-1}\right)\end{array}$ \\
\hline
\end{tabular}

\section{RESULTADOS Y DISCUSIÓN}

Ensayos de bioadsorción en sistema por lotes. Se estudió el efecto del tamaño de partícula evaluando tamaños de 0,355, 0,5 y 1mm, lográndose porcentajes de remoción de $92,89,89,20$ y $77,26 \%$, respectivamente. El mayor porcentaje de adsorción se obtuvo con el tamaño de partícula de $0,355 \mathrm{~mm}$ y esto se puede deber a que al disminuir el tamaño de partícula incrementa el área de contacto y, por tanto, el número de centros activos por unidad de área, que coincide con lo reportado por Han et al. (2016), al usar cáscara de maní, como adsorbente de $\mathrm{Cr}(\mathrm{VI})$ y por Tejada-Tovar et al. (2016b), al emplear biomasas residuales de cáscara de naranja y zuro de maíz, en la remoción de iones de $\mathrm{Pb}$ (II).

El modelo que mejor ajustó los datos experimentales de adsorción de $\mathrm{Cr}(\mathrm{VI})$ sobre biomasa residual de cacao, de acuerdo con la figura 2a, fue el de Freundlich, con un $\mathrm{R}^{2}=0.93$, por lo que durante la adsorción se forman multicapas en la superficie de la biomasa, con una distribución no uniforme de calor y afinidades de adsorción sobre la superficie heterogénea; las moléculas adsorbidas pueden interactuar unas con otras, por la distribución y por la distancia cercana entre los sitios activos de unión, lo cual, permite establecer que la superficie del biomaterial es heterogénea, según lo reportado por Sun-Kou et al. (2014). Además, de acuerdo con los análisis FTIR de las cáscaras de cacao obtenidos en estudios anteriores por Tejada-Tovar et al. (2017), se evidencia la complejidad de la biomasa, debido a la presencia de grupos hidroxilo, carboxilo, fenoles, alifáticos, aminas terciarias y aromáticos, que permiten la interacción entre los centros activos de la biomasa y los iones metálicos. Por otro lado, el valor de la intensidad de adsorción establecida por el parámetro n de la ecuación de Freundlich es de 0,28, en comparación con lo informado por otros trabajos: 0,1376, para el $\mathrm{Pb}$, de acuerdo con Pehlivan et al. (2009) y 1,046, para Ni y 0,60,2 para Zn, obtenido por Calugaru et al. (2016), lo que confirma que el proceso puede ser clasificado como favorable para la adsorción, teniendo en cuenta que el valor de $1 / n$ entre más cercano esté de cero, mayor será la heterogeneidad del adsorbente, según lo reportado por Irani et al. (2011).

Los estudios de la cinética de biosorción son importantes en el tratamiento de efluentes, ya que proporcionan información acerca del mecanismo y la naturaleza del proceso de adsorción; en la figura $2 \mathrm{~b}$, se aprecia que el modelo cinético que mejor ajustó los datos experimentales de adsorción de $\mathrm{Cr}(\mathrm{VI})$ sobre cáscaras de cacao fue el de Elovich, 


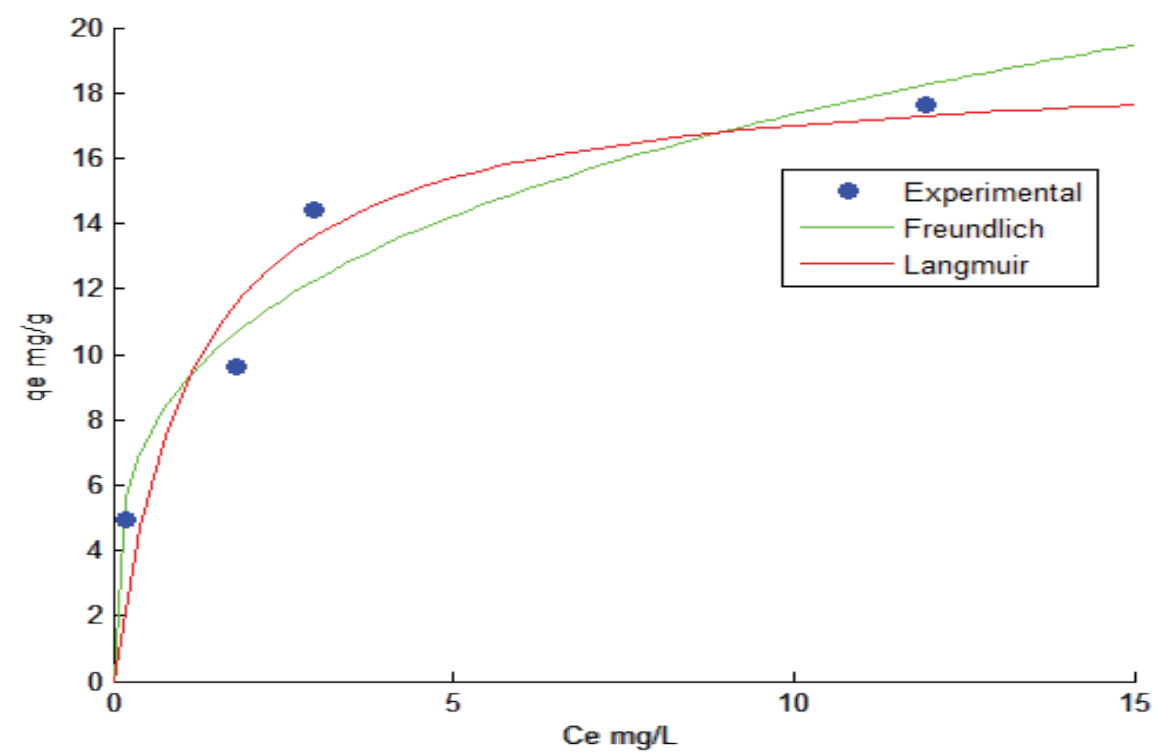

(a)

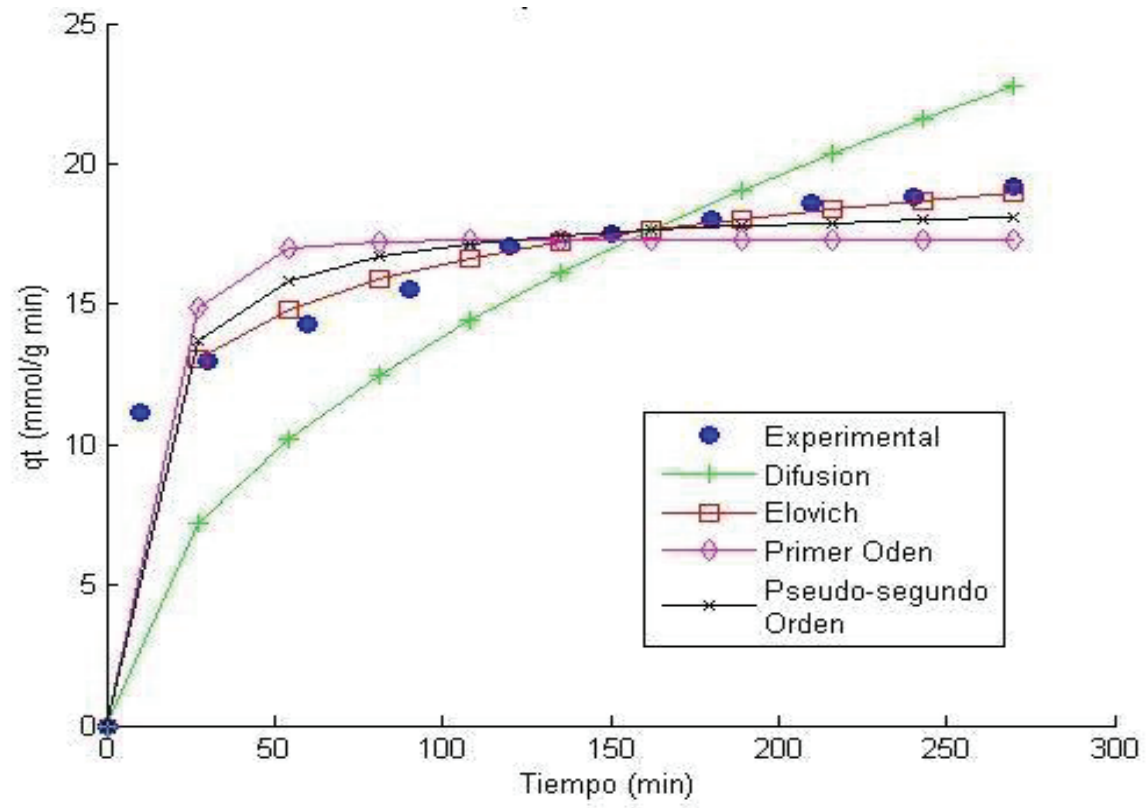

(b)

Figura 2. Ajuste de los datos experimentales de adsorción de $\mathrm{Cr}(\mathrm{VI})$ con cáscara de cacao en sistema batch: (a) los modelos de isotermas de adsorción y (b) los modelos cinéticos.

con $R^{2}=0,97$. Este resultado implica que el mecanismo que controla la adsorción es una reacción de segundo orden y, además, muestra que la superficie catalítica del bioadsorbente es heterogénea, exhibiendo diferentes energías de activación. En este modelo, $\alpha$ se relaciona con la energía de quimiadsorción y $\beta$, con la superficie cubierta, según Figueroa et al. (2004), por lo que se puede decir, que el proceso se produce por quimisorción con centros activos heterogéneos, ya que $\alpha=14,77$; por ello, se exhiben diferentes energías de activación, según lo obtenido por Quiñones et al. (2013) y Tejada-Tovar et al. (2017).

Estudios, como los de Soniya \& Krishnakumar (2015), mostraron que su modelo de adsorción se ajustó al de pseudo segundo orden. También Tejada-Tovar et al. (2015a) hicieron un estudio cinético, removiendo $\mathrm{Cr}(\mathrm{VI})$ en solución con 
cáscara de ñame y bagazo de palma modificados, donde el modelo que mejor describe el proceso es el de Elovich, con una suma de error ${ }^{2}$ de 2,63 y 2,55, para las biomasas, respectivamente.

Se han usado adsorbentes de origen vegetal, tales como fibra de kernel de palma, como lo reportan Kundu et al. (2014); Sargassum filipéndula, por Bertagnolli et al. (2014); cáscara de macadamia, por Pakade et al. (2016); cáscara de cacao modificada con $\mathrm{HCl}$ y $\mathrm{NaOH}$, según Tejada-Tovar et al. (2017); calabaza blanca, de acuerdo con Sreenivas et al. (2014); Pleurotus mutilus, ensayada por Alouache et al. (2017), obteniéndose capacidades de adsorción de $19,1 \mathrm{mg} / \mathrm{g}, \quad 0,819 \mathrm{mmol} / \mathrm{g}, \quad 45,23 \mathrm{mg} / \mathrm{g}, \quad 463,52 \mathrm{mg} / \mathrm{g}$, $76,44 \mathrm{mg} / \mathrm{g}, 76,44 \mathrm{mg} / \mathrm{g}$ y $29,46 \mathrm{mg} / \mathrm{g}$, respectivamente, alcanzándose una capacidad máxima de adsorción en el presente estudio, de $18,98 \mathrm{mg} / \mathrm{g}$, en sistema por lotes.

Ensayos de biosorción en sistema continuo. De los resultados de la adsorción de $\mathrm{Cr}(\mathrm{VI})$ a las condiciones estudiadas para el sistema en continuo, utilizando cáscara de cacao, se encontró que la mayor remoción fue de $39,16 \%$, con el tamaño de partícula de $1,70 \mathrm{~mm}, 30 \mathrm{~g}$ de bioadsorbente y un caudal de $0,75 \mathrm{~mL} / \mathrm{s}$. La diferencia entre los porcentajes de remoción de contaminante obtenidos en sistema por lotes y continuo, se podría explicar, al considerar el uso de tamaños de partícula más grandes, para evitar el taponamiento de la columna, siendo el tamaño de partícula y el caudal variables influyentes en la transferencia de masa; por lo tanto, al tener un mayor diámetro de partículas y menor tiempo de residencia, la remoción se ve disminuida, de acuerdo con lo reportado por Lara et al. (2016).

Se determinó que las condiciones óptimas de operación establecidas para realizar los ensayos de biosorción en sistema continuo son: tamaño de partícula: $1,56 \mathrm{~mm}$; dosis de adsorbente: $46,82 \mathrm{~g}$ y caudal; $0,34 \mathrm{~mL} / \mathrm{s}$. Con esta combinación, los niveles, los cuales maximizan la eficiencia de la remoción, es la empleada en la construcción de la curva de ruptura, con un máximo de adsorción esperado de $62,65 \%$. Posteriormente, se aplicó el RSM a las condiciones óptimas, obteniéndose la ecuación 2 , donde $\mathrm{A}$ es el caudal; $\mathrm{B}$, el tamaño de partícula y $C$, la cantidad de biomasa, con $\mathrm{R}^{2}=0,97$, siendo el modelo de regresión que ajustó los datos experimentales obtenidos del sistema.

$\% R=10.9473-1.36658 \mathrm{~A}-10.2044 \mathrm{~B}+2.45301 \mathrm{C}-0.822804 \mathrm{~A}^{2}+1.87$ $25 A B+1.09 A C+4.49447 B^{2}-2.5925 B C+1.3001 C^{2}$

Debido a limitaciones operacionales, por disponibilidad de tamices y las condiciones del equipo, se usaron como tamaño de partícula $1,7 \mathrm{~mm}$ y un caudal aproximado de $0,3 \mathrm{~mL} / \mathrm{s}$. Posteriormente, se graficaron los datos, obteniéndose el gráfico de superficie respuesta, combinando los tres factores principales y el valor de la variable respuesta en todas las combinaciones resultantes, como se muestra en la figura 3a. A partir del gráfico superficie respuesta (Figura 3a), se corrobora que la variable más influyente en el porcentaje de remoción fue el tamaño de partícula, que afectó de manera positiva. Asimismo, la segunda variable con mayor influencia positiva fue la dosis de adsorbente; por su parte, el caudal influyó negativamente. Por lo anterior, se puede decir que, a menor tamaño de partícula mayor es la remoción y que al aumentar la cantidad de biomasa se vio favorecido el proceso de adsorción.

El MSR se ha usado para optimizar el porcentaje de remoción de cromo en solución usando levadura muerta modificada con nanopartículas de titanio, encontrando que el análisis de varianza mostró un $R^{2}=0,90$ y se obtuvo un modelo de regresión de segundo orden predictivo satisfactorio, teniendo el tiempo de contacto efecto positivo sobre la remoción, mientras el $\mathrm{pH}$ y la concentración inicial de contaminantes efecto negativo, de acuerdo con Choudhury et al. (2017). Asfaram et al. (2015), Dastkhoon et al. (2015) y Dastkhoon et al. (2017) obtuvieron que la contribución de efectos significativos en la cantidad de iones de $\mathrm{Cr}(\mathrm{VI})$ removidos y los efectos con valores absolutos más altos que la línea de referencia, se consideraron estadísticamente significativos en la ecuación polinomial de segundo orden obtenida.

En la tabla 3 aparecen los parámetros calculados de las curvas de ruptura del lecho para los modelos de Adams-Bohart, Thomas y Yoon-Nelson, mientras que en la figura 3b, se observan las gráficas de los modelos predichos y los datos experimentales. Se puede observar que los modelos de Thomas y Yoon-Nelson tiene el mejor ajuste, con $\mathrm{R}^{2}=0,98$, con tendencias similares a los estudios adelantados por Lara et al. (2016). El modelo de Thomas considera que la adsorción no está limitada por la reacción química, sino por la transferencia de masa entre la interface sólido-líquido (AlbisArrieta et al. 2017). El ajuste del modelo de Thomas fue un resultado esperado, debido a que se basa en una cinética de adsorción de segundo orden, al igual que el modelo cinético de Elovich, al que se ajusta el proceso de adsorción de la cáscara de cacao en sistema por lotes, del presente trabajo; además, al ser un modelo más completo que el de YoonNelson, ya que requiere datos relacionados con el adsorbato, el tipo de adsorbente y las propiedades físicas del lecho de adsorción, el modelo cinético de Thomas describe mejor el proceso de adsorción de la cáscara de cacao, lo cual, es coherente con lo informado por Mishra et al. (2016)

Al usar cáscaras de cacao como adsorbente de $\mathrm{Cr}(\mathrm{VI})$ en solución acuosa en sistema por lotes a las condiciones estudiadas, los resultados indicaron que la disminución del tamaño de partícula favorece el proceso de adsorción del metal y que los datos experimentales se ajustan al modelo cinético de 


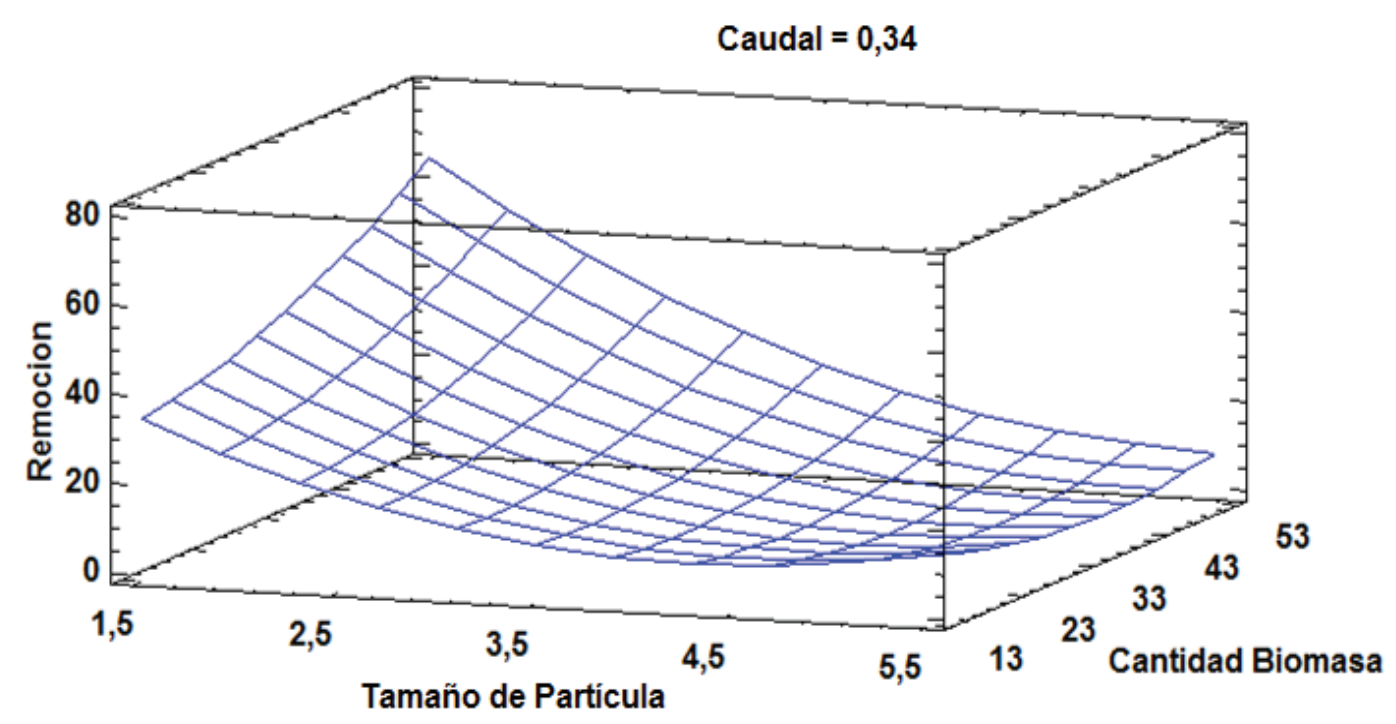

(a)

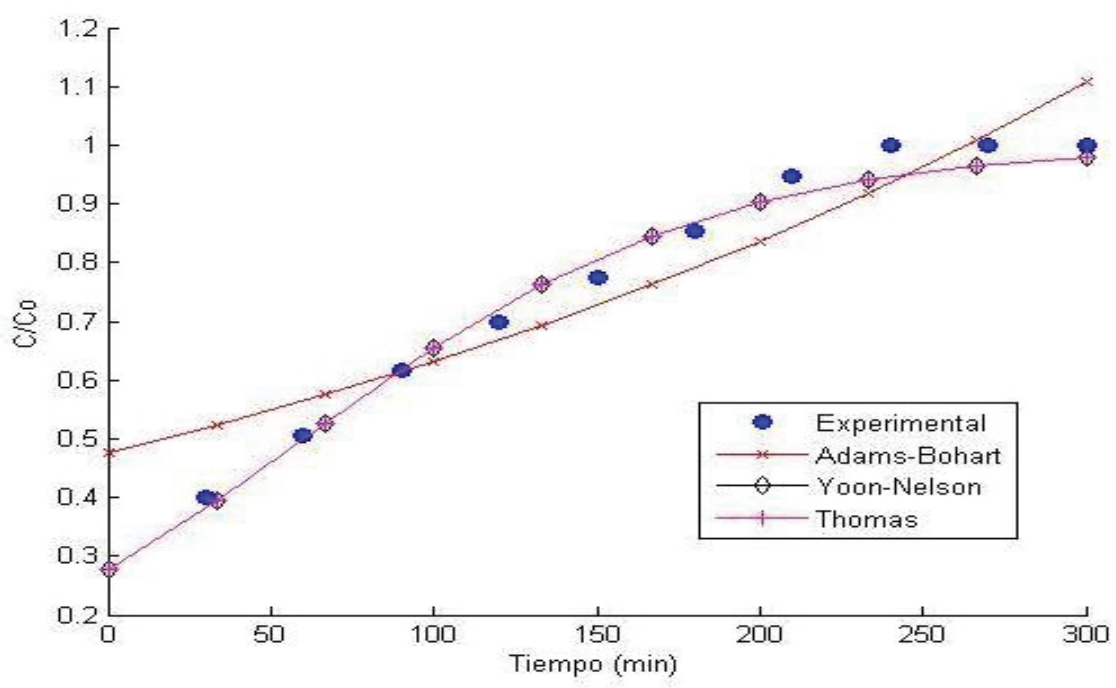

(b)

Figura 3. (a) Superficie de respuesta delas condiciones óptimas del proceso de adsorción de $\mathrm{Cr}(\mathrm{VI})$ sobre cáscaras de cacao en sistema continuo y (b) Curvas de ruptura para la adsorción de $\mathrm{Cr}(\mathrm{VI})$ utilizando cáscara de cacao como bioadsorbente.

Elovich y a la isoterma de Freundlich, con $\mathrm{R}^{2}$ de 0,97 y 0,93, respectivamente, por lo que se establece que la superficie de la cáscara de cacao es heterogénea, con diferentes energías de activación, formándose multicapas en la superficie del adsorbente durante el proceso de adsorción, que el mecanismo que controla el proceso es una reacción de segundo orden y que está controlado por quimiadsorción.

Al realizar experimentos en sistema continuo, usando una columna de lecho empacado, se concluye que las variables más influyentes son el tamaño de partícula y dosis de adsorbente traducida en altura del lecho, estableciendo la metodología superficie de respuesta las condiciones óptimas de adsorción eficientemente, ajustándose los datos experimentales a los modelos cinéticos de Thomas y Yoon-Nelson. Se concluye que la cáscara de cacao es un buen precursor de material bioadsorbente para $\mathrm{Cr}(\mathrm{VI})$.

Para futuras investigaciones, se recomienda evaluar la modificación química y térmica de la biomasa en estudio y probar 
Tabla 3. Valores paramétricos del modelamiento de la Curva de Ruptura con la cáscara de cacao.

\begin{tabular}{|c|c|c|}
\hline \multirow{2}{*}{ Modelo } & \multirow{2}{*}{ Parámetro } & Biomasa \\
\cline { 2 - 3 } & & Cáscara de cacao \\
\hline \multirow{3}{*}{ Adams-Bohart } & $\mathrm{k}_{\mathrm{AB}}$ & 0,00 \\
\cline { 2 - 3 } & $\mathrm{N}_{\mathrm{o}}$ & $3,04 \times 10^{3}$ \\
\cline { 2 - 3 } & $\mathrm{R}^{2}$ & 0,89 \\
\hline \multirow{3}{*}{ Thomas } & $\mathrm{k}_{\mathrm{Th}}$ & 0,003 \\
\cline { 2 - 3 } & $\mathrm{q}_{\mathrm{e}}$ & 128,52 \\
\cline { 2 - 3 } & $\mathrm{R}^{2}$ & 0,98 \\
\hline \multirow{3}{*}{ Yoon-Nelson } & $\mathrm{k}_{\mathrm{N}}$ & 0,02 \\
\cline { 2 - 3 } & & 60,15 \\
\cline { 2 - 3 } & $\mathrm{R}^{2}$ & 0,98 \\
\hline
\end{tabular}

diferentes configuraciones geométricas, que se pueden ver reflejadas en mejor funcionamiento del empaquetamiento de la columna.

Conflictos de intereses: El manuscrito fue preparado y revisado con la participación de todos los autores, quienes declaramos que no existe ningún conflicto de intereses, que ponga en riesgo la validez de los resultados presentados.

\section{BIBLIOGRAFÍA}

1. ALBIS-ARRIETA, A.; ORTIZ TORO, J.; MARTÍNEZ DE LA ROSA, J. 2017. Remoción de cromo hexavalente de soluciones acuosas usando cáscara de yuca (Manihot esculenta): Experimentos en columna. Inge. CUC. 13(1):42-52.

2. AlOUACHE, A.; SELATNIA, A.; HALET, F. 2017. Biosorption of $\mathrm{Cr}(\mathrm{VI})$ from aqueous solutions by dead biomass of Pleurotus mutilus in Torus Reactor. Frontiers in Wastewater Treatment and Modelling. 4:368375.

3. ASFARAM, A.; GHAEDI, M.; HAJATI, S.; GOUDARZI, A.; BAZRAFSHAN, A.A. 2015. Simultaneous ultrasound-assisted ternary adsorption of dyes onto copper-doped zinc sulfide nanoparticles loaded on activated carbon: Optimization by response surface methodology. Spectrochim. Acta A Mol. Biomol. Spectrosc. 145:203-212.

4. BERTAGNOLLI, C.; DA SILVA, M.; GUIBAL, E. 2014. Chromium biosorption using the residue of alginate extraction from Sargassum filipendula. Chem. Eng. J. 237:362-371.
5. CALUGARU, L.; NECULITA, C.; GENTY, T.; BUSSIÈRE, B.; POTVIN, R. 2016. Performance of thermally activated dolomite for the treatment of Niand $\mathrm{Zn}$ in contaminated neutral drain. J. Hazardous Materials. 310:48-55.

6. CHOUDHURY, P.R.; BHATTACHARYA, P.; GHOSH, S.; MAJUMDAR, S.; SAHA, S.; SAHOO, G.C. 2017. Removal of $\mathrm{Cr}(\mathrm{VI})$ by synthesized titania embedded dead yeast nanocomposite: Optimization and modeling by response surface methodology. J. Environ. Chem.-Eng. 5:214-221.

7. CHRISTOFORIDIS, A.K.; ORFANIDIS, S.; PAPAGEORGIOU, S.K.; LAZARIDOU, A.N.; FAVVAS, E.P.; MITROPOLUS, A.CH. 2015. Study of Cu(II) removal by Cystoseira crinitophylla biomass in batch and continuous flow biosorption. Chem. Eng. J. 277:334340.

8. DASTKHOON, M.; GHAEDI, M.; ASFARAM, A.; GOUDARZI, A.; LANGROODI, S.M.; TYAGI, I.; AGARWAL, S.; GUPTA, V.K. 2015. Ultrasound assisted adsorption of malachite green dye onto ZnS:Cu-NP-AC: Equilibrium isotherms and kinetic studies - Response surface optimization. Sep. Purif. Technol. 156:780-788.

9. DASTKHOON, M.; GHAEDI, M.; ASFARAM, A.; GOUDARZI, A.; MOHAMMADI, S.M.; WANG, S. 2017. Improved adsorption performance of nanostructured composite by ultrasonic wave: Optimization through response surface methodology, isotherm and kinetic studies. Ultrason. Sonochem. 37:94-105. 
10. DI NATALE, F.; ERB, A.; LANCIA, A.; MUSMARRA, D. 2015. Equilibrium and dynamic study on hexavalent chromium adsorption onto activated carbon. J. Hazardous Materials. 281:47-55.

11. FEDERACIÓN NACIONAL DE CACAOTEROS -FEDECACAO-. 2016. Economía Nacional: Producción Nacional de cacao en grano (Ton). Disponible desde Internet en: http://www.fedecacao.com.co/portal/ index.php/es/2015-02-12-17-20-59/nacionales (con acceso 21/10/17).

12. FIGUEROA, D.; MORENO, A.; HORMAZA, A. 2004. Equilibrio, termodinámica y modelos cinéticos en la adsorción de rojo 40 sobre tuza de maíz. Ingenierías Universidad de Medellín. 14(26):105-120.

13. HAN, Y.; CAO, X.; OUYANG, X.; SOHI, S.P.; CHEN, J. 2016. Adsorption kinetics of magnetic biochar derived from peanut hull on removal of $\mathrm{Cr}(\mathrm{VI})$ from aqueous solution: Effects of production conditions and particle size. Chemosphere. 145:336-341.

14. IRANI, M.; AMJADI, M.; MOUSAVIAN, M. 2011. Comparative study of lead sorption onto natural perlite, dolomite and diatomite. Chem. Eng. J. 178:317-323.

15. IQBAL, M.; KHERA, R.A. 2015. Adsorption of copper and lead in single and binary metal system onto Fumaria indica biomass. Chem. Intern. 1(3):157b-163b.

16. INYINBOR, A.A.; ADEKOLA, F.A.; OLATUNJI, G.A. 2016. Kinetics, isotherms and thermodynamic modeling of liquid phase adsorption of Rhodamine B dye onto Raphia hookerie fruit epicarp. Water Resour. Ind. 15, 14-27.

17. KUNDU, A.; REDZWAN, G.; SAHU, J.N.; MUKHERJEE, S.; GUPTA, B.S.; HASHIM, M.A. 2014. Equilibrium and dynamic study on hexavalent chromium adsorption onto activated carbon. BioResources. 9(1):14981518.

18. LARA, J.; TEJADA-TOVAR, C.; VILLABONA-ORTÍZ, A.; ARRIETA, A.; GRANADOS-CONDE, C. 2016. Adsorción de plomo y cadmio en sistema continuo de lecho fijo sobre residuos de cacao. Rev. Ion. 29(2):113124.

19. MISHRA, A.; TRIPATHI, B.D.; RAI, A.K. 2016. Packed-bed column biosorption of chromium(VI) and nickel(II) onto fenton modified Hydrilla verticillata dried biomass. Ecotoxicol. Environ. Saf. 132:420-428.
20. MISHRA, S.; BHARAGAVA, R. 2015. Toxic and genotoxic effects of hexavalent chromium in environment and its bioremediation strategies. J. Env. Sci. Health., Part C. 34(1):1-32.

21. NHARINGO, T.; MOYO, M.; MAHAMADI, C. 2016. Kinetics and equilibrium studies on the biosorption of $\mathrm{Cr}(\mathrm{VI})$ by Vigna subterranean (L.) Verdc. hull. Int. J. Environ. Res. 10(1):85-96.

22. PAKADE, V.E.; NTULI, T.D.; OFOMAJA, A.E. 2016. Biosorption of hexavalent chromium from aqueous solutions by Macadamia nutshell powder. Appl. Water Sci. 6:1-16.

23. PEHLIVAN, E.; OZKAN, A.; DINC, S.; PARLAYICI, S. 2009. Adsorption of $\mathrm{Cu}^{2+}$ and $\mathrm{Pb}^{2+}$ ion on dolomite powder. J. Hazardous Materials. 167:1044-1049.

24. QUIÑONES, E.; TEJADA-TOVAR, C.; ARCIA, C.; RUIZ, V. 2013. Remoción de plomo y níquel en soluciones acuosas usando biomasas lignocelulósicas: una revisión. Rev. U.D.C.A Act. \& Div. Cient. 16(2):479-489.

25. SINGH, A.; PAL, R.; GANGWAR, C.; GUPTA, A.; TRIPATHI, A. 2015. Release of heavy metals from industrial waste and e-waste burning and its effect on human health and environment. Intern. J. Emerging Res. Manag. Tech. 4(12):51-56.

26. SONG, S.T.; HAU, Y.F.; SAMAN, N.; JOHARI, K.; CHEU, S.C.; KONG, H.; MAT, H. 2016. Process analysis of mercury adsorption onto chemically modified rice straw in a fixed-bed adsorber. J. Environ. Chem. Eng. 4(2):1685-1697.

27. SONIYA, M.; KRISHNAKUMAR, G. 2015. Biosorption of heavy metals from aqueous solution using mangrove fern Acrostichum aureum L. leaf biomass as a sorbent. Int. Res. J. Environment Sci. 4(11):25-31.

28. SREENIVAS, K.M.; INAKAR, M.B.; GOKHALE, S.V.; LELE, S.S. 2014. Re-utilization of ash gourd (Benincasa hispida) peel waste for chromium (VI) biosorption: Equilibrium and column studies. J. Environ. Chem. Eng. 2(1):455-472.

29. SUIN-KOU, M.; OBREGÓN-VALENCIA, D.; PINEDOFLORES, Á.; PAREDES-DOIG, A.; AYLAS-OREJÓN, J. 2014. Adsorción de metales pesados empleando carbones activados preparados a partir de semillas de aguaje. Rev. Soc. Quim. Perú. 80(4):225-236. 
30. TAGHIPOUR, M.; JALALI, M. 2015. Effect of clay minerals y nanoparticles on chromium fractionation in soil contaminated with leather factory waste. J. Hazardous Materials. 297:127-133.

31. TEJADA-TOVAR, C.; VILLABONA-ORTÍZ, A., GONZÁLEZ-DELGADO, A.D.; MARRUGO-CANTILLO, E.; PÁJARO-MONTERO, M. 2018. Effect of Bed Height and Biomass Array on Removal of an Anion Surfactant Using a Continuous Rapid-Mixed Biofilter. Contemp. Eng. Sci. 11(7):297-305.

32. TEJADA-TOVAR, C.; VILLABONA-ORTÍZ, A.; JIMÉNEZ, M. 2017a. Remoción de cromo hevavalente sobre residuos de cacao pretratados químicamente. Rev. U.D.C.A. Act. \& Div. Cient. 20(1):139-147.

33. TEJADA-TOVAR, T; VILLABONA-ORTIZ, A.; ALVAREZBAJAIRE, G.; JATTIN-TORRES, L.; GRANADOSCONDE, C. 2017b. Influencia de la altura del lecho sobre el comportamiento dinámico de columna de lecho fijo en la biosorción de mercurio. Revista Tecno Lógicas, 20(40):71-81.

34. TEJADA-TOVAR, C.; HERRERA, A.; RUIZ, E. 2016a. Kinetic and isotherms of biosorption of $\mathrm{Hg}$ (II) using citric acid treated residual materials. Ingeniería y competitividad. 18(1):117-127.

35. TEJADA-TOVAR, C.; HERRERA, A.; NÚÑ̃EZ-ZARUR, J. 2016b. Remoción de plomo por biomasas residuales de cáscara de naranja (Citrus sinensis) y zuro de maíz (Zea mays). Rev. U.D.C.A. Act. \& Div. Cient. 19(1):169-178.

36. TEJADA-TOVAR, C.; HERRERA, A.; RUIZ-PATERNINA, E. 2016c. Adsorción de plomo y cadmio en sistema continuo de lecho fijo sobre residuos de cacao. Rev. Ion. 29:111-122.

37. TEJADA-TOVAR, C.; VILLABONA-ORTíZ, A.; GARCÉS, L. 2015a. Adsorción de metales pesados en aguas residuales usando materiales de origen biológico. Tecno Lógicas. 18(34):109-123.
38. TEJADA-TOVAR, C.; HERRERA, A.; NÚÑ̃EZ, J.R. 2015b. Adsorción competitiva de Ni (II) y Pb (II) sobre materiales residuales lignocelulósicos. Investigaciones Andina. 18(31):1355-1367.

39. TEJADA-TOVAR, C.; VILLABONA-ORTÍZ, A.; GARCÉSJARABA, L.E. 2015c. Kinetics of adsorption in mercury removal using cassava (Manhiot esculenta) and lemon (Citrus limonum) wastes modified with citric acid. Ingeniería y Universidad. 19(2):283-298.

40. TEJADA-TOVAR, C.; QUIÑONEZ-BOLAÑOS, E.; TEJEDA-BENITEZ, L.; MARIMÓN-BOLÍVAR, W. 2015d. Absorción de Cromo Hexavalente en soluciones acuosas por cáscaras de naranja (Citrus sinensis). Producción + Limpia. 10(1):9-21.

41. TEJADA-TOVAR, C.; VILLABONA-ORTÍZ, A.; PATERNINA-ORTÍZ, E. 2015e. Cinética de adsorción de $\mathrm{Cr}$ (VI) usando biomasas residuales modificadas químicamente en sistemas por lotes y continuo. Rev. Ion. 28(1):29-41.

42. VIJAYARAGHAVAN, K.; RANGABHASHIYAM, S.; ASHOKKUMAR, T.; AROCKIARAJ, J. 2016. Monoand multi-component biosorption of lead(II), cadmium(II), copper(II) and nickel(II) ions onto cocopeat biomass. Sep. Sci. Techol. 51(17):2725-2733.

43. WU, Y.; LUO, H.; WANG, H.; WANG, C.; ZHANG, J.; ZHANG, Z. 2013. Adsorption of hexavalent chromium from aqueous solutions by graphene modified with cetyltrimethylammonium bromide. J. Colloid Interface Sci. 394:183-191.

Recibido: Agosto 28 de 2017

Aceptado: Marzo 28 de 2018

Cómo citar:

Tejada-Tovar, C.; Villabona-Ortíz, Á.; Caballero Romero, V.; Paternina Cuesta, J.; Granados Conde, C. 2018. Optimización de parámetros para la construcción de la curva de ruptura en la adsorción de $\mathrm{Cr}(\mathrm{VI})$ sobre cáscara de cacao. Rev. U.D.C.A Act. \& Div. Cien.21(1): 167-177. 